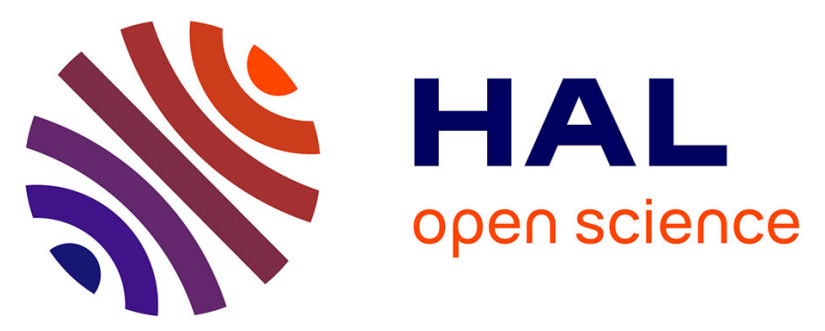

\title{
Oxygen concentration and modeling thermal decomposition of a high-performance material: A case study of polyimide (Cirlex)
}

Aditya Ramgobin, Gaelle Fontaine, Serge Bourbigot

\section{- To cite this version:}

Aditya Ramgobin, Gaelle Fontaine, Serge Bourbigot. Oxygen concentration and modeling thermal decomposition of a high-performance material: A case study of polyimide (Cirlex). Polymers for Advanced Technologies, In press, 10.1002/pat.5060 . hal-02938129

HAL Id: hal-02938129

https://hal.univ-lille.fr/hal-02938129

Submitted on 14 Sep 2020

HAL is a multi-disciplinary open access archive for the deposit and dissemination of scientific research documents, whether they are published or not. The documents may come from teaching and research institutions in France or abroad, or from public or private research centers.
L'archive ouverte pluridisciplinaire $\mathbf{H A L}$, est destinée au dépôt et à la diffusion de documents scientifiques de niveau recherche, publiés ou non, émanant des établissements d'enseignement et de recherche français ou étrangers, des laboratoires publics ou privés. 
2 Thermal Decomposition of a High-

3 Performance Material: A Case Study of

4 Polyimide (Cirlex)

5 Aditya Ramgobin ${ }^{1}$, Gaëlle Fontaine ${ }^{2}$ and Serge Bourbigot ${ }^{2 *}$

61 Univ. Lille, CNRS, INRAE, Centrale Lille, UMR 8207 - UMET - Unité Matériaux et

7 Transformations, F-59000 Lille, France, aditya.ramgobin@univ-lille.fr,

82 Univ. Lille, CNRS, INRAE, Centrale Lille, UMR 8207 - UMET - Unité Matériaux et

9 Transformations, F-59000 Lille, France, gaelle.fontaine@ensc-lille.fr

10 * Correspondence: serge.bourbigot@ensc-lille.fr

11 Abstract: Kinetic decomposition models for the thermal decomposition of a highperformance polymeric material (Polyimide, $\mathrm{Pl}$ ) were determined from specific techniques. Experimental data from thermogravimetric analysis (TGA) and previously elucidated decomposition mechanism were combined with numerical simulating tool to establish a comprehensive kinetic model for the decomposition of PI under three atmospheres: nitrogen, $2 \%$ oxygen, and synthetic air. Multi-staged kinetic models with subsequent and competitive reactions were established by taking into consideration the different types of reactions that may be occurring during the thermal decomposition of the material (chain scission, thermo-oxidation, char formation). The decomposition products and decomposition mechanism of PI which was established in our previous report allowed for the elucidation of the kinetic decomposition models. A three-staged kinetic thermal decomposition pathway was a good fit to model the thermal decomposition of PI under nitrogen. The kinetic model involved an autocatalytic type of reaction followed by successive nth order reactions. Such types of models were set up for the evaluation of the kinetics of the thermal decomposition of $\mathrm{PI}$ under $2 \%$ oxygen and in air, leading to models with satisfactory fidelity.

Keywords: kinetic analysis, polymer decomposition, high performance polymers, simulation 
31 Polyimide (Cirlex or Kapton, $\mathrm{PI}$ ) is a high-performance polymeric material used in a 32 wide array of applications, due to its outstanding physical properties. Indeed, 33 polyimides are known to have exceptional thermal stability. This is attributed to their 34 rigid heterocyclic imide and aromatic rings on the polymer backbone ${ }^{1}$. They are used 35 in fields such as microelectronics, high temperature matrices, as well as for gas 36 separation membranes.

37 Previous work on the thermal stability and decomposition of polyimide has been 38 performed at our laboratory ${ }^{2}$, whereby different behavioral traits of $\mathrm{PI}$ were 39 investigated. These traits involved the investigation of the thermal decomposition 40 mechanism under pyrolytic conditions, the incidence of oxygen concentration on the 41 thermal stability of $\mathrm{PI}$, as well as the evaluation of the fire behavior of $\mathrm{PI}$ under 42 different heat fluxes using the mass loss cone calorimeter.

43 In our previous work, we have evidenced that the thermal behavior of $\mathrm{PI}$ is quite 44 different depending on the oxygen concentration. There are several stages involved 45 in the thermal decomposition of $\mathrm{PI}$, and the stages differ greatly whether the thermal 46 stress is oxygen rich or deprived. Therefore, in order to complement the thermal 47 decomposition of PEEK in a fire scenario, kinetic models corresponding the 48 decomposition in oxygen-free, oxygen-poor, and oxygen rich (air) atmosphere need 49 to be investigated.

50 The TG and DTG plots of thermogravimetric analysis (TGA) performed on PI at 51 different oxygen concentrations (Figure 1) show that the initial degradation 52 temperature is not highly dependent on the oxygen concentration. However, it is 53 clear from the TG plots that at high temperatures, the role of oxygen in the thermal 54 decomposition of $\mathrm{PI}$ is significant. Indeed, at higher temperatures (above $600{ }^{\circ} \mathrm{C}$ ), 55 the mass loss increases with increasing oxygen concentration. This suggests that 56 the thermal decomposition mechanism is also highly dependent on the oxygen 57 concentration, even if it is as low as $2 \%$.

Figure 1. TG and DTG plots of PI heated at $10{ }^{\circ} \mathrm{C} / \mathrm{min}$ under nitrogen, $2 \%, 4 \%, 8 \%$ and $12 \%$, and in air ${ }^{2}$.

60 In order to further the understanding of the thermal decomposition characteristics of $61 \mathrm{PI}$, it was undeniably important to evaluate the kinetic parameters that govern its thermal decomposition. In our previous work, we have observed that the presence 63 of oxygen may impact both the onset of the thermal decomposition and the char 64 degradation process ${ }^{2}$.

65 Different oxygen concentrations correspond to different parts of a fire scenario. 66 Indeed, a material under a flame is scarcely in contact with oxygen because the 67 flame usually consumes all the oxygen in the surrounding environment. Therefore, 68 the decomposition behavior at this point corresponds to solely pyrolytic effects. 
However, if there is the presence of only a small flame, there may be some oxygen (much lowered concentration as compared to air, as low as $2 \%$ ) that is in contact with the material. Furthermore, if the material is found away from a flame in a wellventilated environment, it subjected to thermal stress in the presence of air. Thus, modelling the three oxygen levels may provide an integral insight on the decomposition behavior of a burning material.

Therefore, it was of interest to evaluate the kinetic parameters of the thermal decomposition of the material under three different oxygen concentrations: $0 \%, 2 \%$, and $20 \%$ (synthetic air).

\section{Materials and Methods}

Polyimide (Cirlex CL) was purchased from Goodfellow Cambridge Limited (Huntington, England)

\subsection{Thermogravimetric Analysis (TGA)}

Thermogravimetric analyses (TGA) were conducted on a Netzsch Libra instrument. Powdered samples of $9-10 \mathrm{mg}$ (according to the good practice of TGA, it is assumed the samples are thermally thin) were placed in open alumina pans and heated up to $900^{\circ} \mathrm{C}$ under different percentages of oxygen and nitrogen at different heating rates $\left(1,2,5\right.$, and $\left.10^{\circ} \mathrm{C} / \mathrm{min}\right)$ up to $900{ }^{\circ} \mathrm{C}$ under $0 \%, 2 \%$, and $20 \%$ oxygen concentrations.

\subsection{Kinetic Analysis}

Kinetic analysis and modeling of the degradation of the samples were made using a Kinetics Neo software package developed by Netzsch Company. The principle has been discussed by Opfermann in ${ }^{3}$ and here we only briefly remind the reader of the basic concepts of the method.

For kinetic analysis, it is assumed that the material decomposes according to Eq. (1):

$$
\mathrm{A}_{\text {solid }} \rightarrow \mathrm{B}_{\text {solid or liquid }}+\mathrm{C}_{\text {gas }}
$$

The rate expression $\mathrm{de} / \mathrm{d} t$, where $e$ is the concentration of educt (reactant), is assumed to be defined by Eq. (2):

$$
\frac{\mathrm{de}}{\mathrm{dt}}=\mathrm{k}(\mathrm{T}) \times \mathrm{f}(\mathrm{e}, \mathrm{p})
$$

Where $\mathrm{k}$ is the kinetic constant, $\mathrm{p}$ is the concentration of the product, $k=A \exp$ ($E / R T)$ according to the Arrhenius law, $A$ is the frequency factor, $E$ is the activation energy and $f(e, p)$ is the so-called "reaction equation" or in the case of TGA, the "reaction model". 
All reactions are assumed to be irreversible. In the case of degradation and since the evolved gases are continuously removed by the fluid flow in the TGA chamber, this is a reasonable assumption. It is also assumed that the overall reaction (Eq. (9)) is the sum of individual reaction stages (formal or true stage) with constant activation energy, as generally accepted in chemistry. The model can then include competitive, independent and successive reactions. The equations are solved with multivariate kinetic analysis (determination of the parameter via a hybrid normalized GaussNewton method or Marquardt method) ${ }^{4}$.

By optimizing the models used for the kinetic analysis, the kinetic parameters of each stage in the thermal decomposition model of the material can be evaluated, allowing for a better understanding of the thermal decomposition behavior of the material.

The approach used to model the decomposition behavior of PEEK was similar to that adopted by Moukhina et al, whereby a model free analysis was used to determine the initial kinetic decomposition parameters and gain insight regarding the number of stages involved and the types of kinetic models that govern the decomposition ${ }^{5}$. To this approach, we added our own understanding of the thermal decomposition based our previous work on the same material ${ }^{2}$, whereby the thermal decomposition mechanism was attempted.

The reflection behind the methodology that we have adopted is briefly summarized hereunder.

In order to model the kinetic degradation of a polymeric material, two separate functions can be assumed. One being temperature dependent $(K(T))$, and the other governed by the conversion, $\alpha, f(\alpha)$. The latter can be of any value from 0 (no degradation) to 1 (complete degradation). Therefore, the differential equation that defines the kinetics of thermal degradation can be written as equation $3^{6}$.

$$
\frac{d \alpha}{d t}=K(T) f(\alpha)
$$

$\frac{d \alpha}{d t}$ is the rate of degradation, $\mathrm{K}(\mathrm{T})$ is the temperature dependent rate constant, and $f(\alpha)$ corresponds to the reaction model. $K(T)$ can be described by the Arrhenius equation (equation 4):

$$
K(T)=A e^{-\left(\frac{E}{R T}\right)}
$$

Where $\mathrm{R}$ is the universal gas constant, $E$, the activation energy, and $A$, the preexponential factor ${ }^{7}$. 
The time dependence of equation (4) can be eliminated by using a constant heating rate $\beta=\frac{d T}{d t}$, by dividing by it (equation 5 ).

$$
\frac{d \alpha}{d T}=\frac{A}{\beta} f(\alpha) e^{-\frac{E}{R T}}
$$

138

Linearizing equation 5 leads to obtainable kinetic parameters ( $A$ and $E$ ) by using the equation 6.

$$
\ln \left(\frac{\frac{d \alpha}{d T}}{f(\alpha)}\right)=\ln \left(\frac{A}{\beta}\right)-\left(\frac{E}{R T}\right)
$$

One approach for kinetic modelling involved the assumption that the activation energy and the preexponential factor are constant. A well-known technique that uses this method is the Friedman method, whereby the activation energy and preexponential factor are obtained by plotting the logarithmic form of the rate equation of each heating rate (equation 3). $\alpha$ represents the value at a certain degree of conversion, and $i$ the data from the corresponding heating rate experiment ${ }^{8}$.

$$
\left.\ln \left[\beta_{i}\left(\frac{d \alpha}{d T}\right)_{\alpha, i}\right]=\ln \left(A_{\alpha} f(\alpha)\right)-\frac{E_{\alpha}}{R T_{\alpha, i}}\right)
$$

The activation energy at particular conversion degrees can be calculated with linear regression from a plot of $\ln \left[\beta_{i}\left(\frac{d \alpha}{d T}\right)_{\alpha, i}\right]$ against $\frac{1}{T_{\alpha, i}}$ for the heating rates that were used. The plot can provide confirmation as to whether there is more than one stage involved in the degradation process. Moreover, the nature of the decomposition stage can also be deduced by comparing the slope of a constant heating rate data 9 . By comparing the magnitudes at the peak slope (the one that is on the right side of the peak) and that of isoconversion lines, three types of reactions are defined: normal, accelerated, and retarded (Figure 2).

Figure 2. Friedman plots corresponding to a normal type of reaction (left), an accelerated reaction (middle), and a retarded reaction (right). ${ }^{9}$

A normal reaction corresponds to the curve whereby the magnitude of the peak slope (slope to the right of the peak) and that of the isoconversion lines are of the same magnitude. An accelerated reaction is one whereby the peak slope is steeper than that of the isoconversion lines. Contrarily, a retarded reaction has a peak slope which is gentler than that of the isoconversion lines. However, one of the major limitations of this method is that it does not cater for the possibility of competitive parallel reactions that may occur during the thermal decomposition process. 
It should be noted that for the reaction model to make physical sense, reaction orders above three are not considered. However, because of the complexity of the reactions occurring during the thermal decomposition of a polymeric material, optimizations based on experimental data can lead to non-integer values of reaction orders. This often happens when a stage involves more than one pathway towards the same decomposition product.

171 Another similar method that is used for model free analyses of kinetic degradation is the Ozawa-Flynn-Wall integral isoconversional method ${ }^{10,11}$.

173 The Ozawa-Flynn-Wall analysis involves an integral method for the calculation of the 174 kinetic parameters, therefore, there is no separation of variables involved. As a result, competitive reactions show variations in activation energies between the Ozawa-Flynn-Wall and the Friedman analyses ${ }^{9}$. This will be helpful in determining the nature of the stages involved in the thermal decomposition of the materials investigated.

Moreover, the insight regarding the thermal decomposition behavior of the polymeric materials will be used in order to devise an experiment-based model, aided by the model free analysis detailed above.

There are several reaction types that can be attributed to a decomposition stage. The typical homogenous reactions and classic solid reactions are listed in Table 1.

The models were optimized using the KineticsNeo software (Netzsch). We have attempted to make kinetic models with the lowest number of stages that gave an acceptable fit and that were consistent with the thermal decomposition mechanism of the material studied.

Table 1. Reaction types and corresponding reaction equations $\frac{d \alpha}{d t}=-A e^{\frac{E}{R T}} f(\alpha)$

\section{Results and Discussion}

\subsection{Pl decomposition in nitrogen}

191 TG curves and their corresponding DTG curves when PI is subjected to four different heating rates under nitrogen are shown on Figure 3.

Figure 3. TGA (left) and the corresponding DTG (right) curve of PI at 1 (black), 2 (red), 5 (blue), and $10 \mathrm{~K} / \mathrm{min}$ (magenta), under nitrogen.

195 From the TGA plots of $\mathrm{PI}$ (Figure 3), it is clear that PI has a high thermal stability as hardly any mass loss is recorded until almost $500^{\circ} \mathrm{C}$. he temperature at the onset of the decomposition increases with increasing heating rate. It involves an apparent sharp decomposition stage, whereby around $30 \mathrm{wt} \%$ of mass loss is observed. This 
mass loss rate decreases, but only to around $0.5 \mathrm{wt} \% /{ }^{\circ} \mathrm{C}$. This suggests that there is another stage involved in the thermal decomposition of $\mathrm{PI}$.

202 It can be noted that the maximum mass loss rate decreases with increasing heating rate. This suggests that the first decomposition stage follows the same kinetic pathway. To further the understanding of the kinetics of the thermal decomposition behavior, the model free analysis (Friedman analysis) was performed on the TGA curves of $\mathrm{Pl}$ at the different heating rates. The Friedman plot as well as the isoconversion lines corresponding to different conversions are plotted on Figure 4.

Figure 4. Friedman analysis of $\mathrm{PI}$ using 4 different heating rates under nitrogen (orange: $1 \mathrm{~K} / \mathrm{min}$, green: $2 \mathrm{~K} / \mathrm{min}$, red: $5 \mathrm{~K} / \mathrm{min}$, blue: $10 \mathrm{~K} / \mathrm{min}$ )

210 From the Friedman analysis (Figure 4), it can be observed that the isoconversion 211 lines to the right of the first peak has a gentler slope than the slope of the peaks. 212 This means that the first decomposition reaction is accelerated. This is often the case in polymeric materials because their decomposition leads to the generation of radicals which are very reactive and contribute to the further decomposition of the material. For this reason, the first stage of the thermal decomposition was assigned to an autocatalytic decomposition rate model $(\mathrm{Cn})$.

217 Moreover, the activation energy and pre-exponential factor based on the model free Friedman analysis is shown on Figure 5.

Figure 5. Activation energy plot for PI under nitrogen, obtained by Friedman analysis

From the two plots on Figure 5, it can be seen that the activation energy and the pre-exponential factor are relatively constant at the beginning of the decomposition process, until around $60 \%$ of conversion. After this, the activation energy increases rapidly from around 300 to around $1400 \mathrm{~kJ} / \mathrm{mol}$ at $100 \%$ conversion. This means that there are at least two stages in the thermal decomposition of PI under nitrogen. Moreover, at around $90 \%$ conversion, there seems to be a slight change in the continuity of the curve, suggesting that there might be another step in the decomposition reaction. In order to check whether there are any competitive reactions occurring, the activation energies calculated from an Ozawa-Flynn-Wall curve was plotted on the same axes as the activation energies from the Friedman plot. This graph is shown on Figure 6.

Figure 6. Activation energies calculated using Friedman analysis (black) and the Ozawa-Flynn-Wall method (magenta) for PI under nitrogen

233 By comparing the evolution of the activation energies calculated from the two 234 aforementioned methods, it can be seen that for the first $50 \%$ of the conversion, there is very little difference in activation energy. This means that there are not 
competitive reactions in the first step of the thermal decomposition. However, at higher conversions, we can see that the activation energies curves do not have the exact same shape, but the values are only slightly different.

From the model free analyses, we have seen that there are at least three stages in the thermal decomposition of PI under nitrogen. Additionally, the comparison of the activation energies from the derivative model free analysis (Friedman) and the integral model free analysis (Ozawa-Flynn-Wall), we have deduced that there are not competitive reactions occurring during the thermal decomposition. Moreover, it was seen from both the activation energy plots that towards the end of the conversion, the activation energy of the thermal decomposition increases greatly.

These inputs are essential for the elaboration of a kinetic model for the thermal decomposition mechanism of PI. The model that was chosen is illustrated on Scheme 1 and explained in the subsequent paragraphs.

Scheme 1. Kinetic model used to model the thermal decomposition kinetics of PI under nitrogen

Moreover, in the mechanism of the thermal decomposition of PI that was detailed in our previous work ${ }^{2}$, the different reactions occurring during the thermal breakdown of PI under nitrogen are also known. The first stage of the decomposition involves the random scission of the polymer chain. This corresponds to the first decomposition stage, which is also the main decomposition stage of the thermal decomposition. The kinetic model that was used for this stage of the decomposition corresponds to an autocatalytic reaction. Indeed, the random scission of a polymeric chain leads to the formation of reactive radicals that often take part in the subsequent decomposition of a material.

After the random scission of the polymeric chains, small amounts of gases such as ammonia, hydrogen cyanide, and methane gave off and were characterized by TGA connected to Fourier Transform Infrared (FTIR). The formation of methane and hydrogen cyanide probably comes from the secondary decomposition of aromatics that are formed during the char formation process. Indeed, at high temperatures, the char continues to strengthen and degrade, releasing hydrogen and low molecular weight products such as methane. This corresponds to part of the second stage of the decomposition of $\mathrm{PI}$ under nitrogen.

267 Furthermore, the presence of hydrogen (which is assumed by the presence of methane), means that nitrogen containing moieties may be reduced to ammonia at high temperatures. This explains the slightly delayed detection of ammonia in the TGA-FTIR ${ }^{2}$, and corresponds to the third stage. Table 2 shows the kinetic parameters that were optimized for the decomposition model. 
The last two stages correspond to extremely high activation energies, which is coherent with both the Friedman and the Ozawa-Flynn-Wall methods. However, the exact reactions occurring at such high temperatures are unknown. This is because the exact structure of the char formed during the initial decomposition is not perfectly known. However, we can safely mention that the 3-stage reaction is coherent with the release of the decomposition products that were identified.

The first stage of the decomposition was assigned to an autocatalytic decomposition, with an activation energy around $278 \mathrm{~kJ} / \mathrm{mol}$, a reaction order of 3.00 and an autocatalytic order of 0.377 . This activation energy is coherent with the Friedman analysis Figure 5. The two subsequent reactions were modeled as Arrhenius reactions, with activation energies of 370 and $410 \mathrm{~kJ} / \mathrm{mol}$, respectively. This corresponds to the high activation energy seen for the decomposition at conversions superior to $60 \%$. These two reactions correspond to the slow decomposition of the char formed during the first stage of the decomposition and each share around $20 \%$ of contribution to the whole mechanism.

Moreover, from Table 2, it can be seen that the first decomposition stage, which corresponds to the autocatalytic stage, is the one that has the highest contribution. The other two stages are also kinetically significant, with contributions of around $19 \%$ From the kinetic parameters in Table 2, the best fit model of mass loss with temperature was plotted along with the experimental TGA curves. The resulting graph is shown on

\section{Figure 7.}

Figure 7. Best fit of the TG data for the three-stage reaction models in Scheme 1, with the parameters given on Scheme 1. The curves represent the experimental data and the symbols represent the model

From a statistical point of view, the correlation coefficient of the model with respect to the experimental data was 0.99964 .

From the model that was adopted, the major decomposition reaction corresponds to the first stage, with a contribution of $62.5 \%$ in the model. However, the subsequent stages have a non-negligible contribution (18.8\%) to the model. This means that the char decomposition plays a major role in the thermal decomposition model of $\mathrm{PI}$.

Having the kinetics of the decomposition of PI under nitrogen in hand, our interest went to the effect that oxygen may have on kinetics of the thermal decomposition of $\mathrm{PI}$. Therefore, the thermal behavior of $\mathrm{PI}$ under $2 \%$ oxygen was studied and is detailed in the next section.

\subsection{PI decomposition in $2 \%$ oxygen}


To model the kinetic decomposition of PI under $2 \%$ oxygen, dynamic TGA was performed on $\mathrm{PI}$ at low oxygen levels at different heating rates. The resulting TG curves and their corresponding DTG curves of PI under $2 \%$ oxygen are shown on Figure 8.

Figure 8. TGA (left) and the corresponding DTG (right) curve of PI at 1 (black), 2 (red) ,5 (blue), and $10 \mathrm{~K} / \mathrm{min}$ (magenta), under $2 \%$ oxygen

Quite expectedly and similarly to the initial investigation of PI under different oxygen levels, PI remains stable up to a relatively high temperature of around $450{ }^{\circ} \mathrm{C}$. From the TG curves it appears that there is only one decomposition stage in the thermal decomposition of $\mathrm{PI}$ under $2 \%$ oxygen. Nevertheless, when looking at the DTG curves for heating rates corresponding to 2,5 , and $10 \mathrm{~K} / \mathrm{min}$, there seems to be at least two peaks for the thermal decomposition of $\mathrm{PI}$. The absence of a visible second peak at the heating rate of $1 \mathrm{~K} / \mathrm{min}$ is assigned to the two decomposition stages occurring at similar temperature ranges.

Therefore, from the TGA and DTG curves, we can deduce that the thermal decomposition mechanism of $\mathrm{Pl}$ is at least a two-stage process. To confirm this, Friedman analysis was performed on the data from the above TG curves. The resulting plot is shown in Figure 9.

Figure 9. Friedman analysis of PI using 4 different heating rates under $2 \%$ oxygen (orange: $1 \mathrm{~K} / \mathrm{min}$, green: $2 \mathrm{~K} / \mathrm{min}$, red: $5 \mathrm{~K} / \mathrm{min}$, blue: $10 \mathrm{~K} / \mathrm{min}$ )

The Friedman plot shows a small peak at the beginning of the decomposition, followed by another at around $50 \%$ conversion. Finally, another decomposition stage is visible towards $100 \%$ conversion. This peak is not visible in the Friedman plot at $10 \mathrm{~K} / \mathrm{min}$. This may be because at the maximum temperature of the TG experiment, the mass loss was not total, suggesting that the reaction had not reached its completion. From these plots, we can deduce that there are at least three stages involved in the thermal decomposition of PI under $2 \%$ oxygen.

From this Friedman plot, the activation energies as well as the pre-exponential factor for the decomposition of PI was plotted with respect to the conversion (Figure 10). This is essential to obtain initial parameters for the modeling of the thermal decomposition of $\mathrm{PI}$.

Figure 10. Activation energy plot for PI under $2 \%$ oxygen, obtained by Friedman analysis

From this Friedman plot, it can be seen that at low conversion, the activation energy is relatively high (around $350 \mathrm{~kJ} / \mathrm{mol}$ ). This corresponds to the first stage of the decomposition. However, as the material decomposes further, the activation energy decreases rapidly and is dependent on the conversion degree. This is assigned to the second stage of the thermal decomposition. At conversions above $70 \%$, the 
activation energy is still dependent on the conversion, but unlike the previous stage, it seems to increase slightly. This is assigned to the third stage of the decomposition.

347 Based on the two Friedman analysis plots, we have seen that the thermal decomposition of $\mathrm{PI}$ under $2 \%$ oxygen corresponds to at least three stages of decomposition. In order to have insight concerning eventual competitive reactions that might be occurring during the decomposition of $\mathrm{PI}$, the activation energy based on calculations using Friedman analysis was compared to that based on the OFW method (Figure 11). Because of the difference in the approach for the two similar methods, the occurrence of competitive reactions is translated by a difference in activation energy for the same conversion.

Figure 11. Activation energies calculated using Friedman analysis (black) and the Ozawa-Flynn-Wall method (magenta) for PI under $2 \%$ oxygen

From the two activation energy plots, it can be seen that the global shapes of the two activation energy plots are relatively similar. Indeed, the activation energy increases for early conversions and then decreases. The increase in activation energy can be assigned to the breaking of chemical bonds (endothermic) which generate radicals. The following decrease in activation energy is assigned to recombination reactions between radicals which potentially lead to the formation of a stable char (exothermic).

Moreover, there is a slight difference in the activation energy for the first stage of decomposition. However, the error is significant for the first stage of the decomposition. It is therefore hard to conclude whether the competitive stage occurs during the first stage or later. As a result, the kinetic decomposition model was based on the understanding of chemical reactions occurring at the onset of the decomposition.

371 The model free analyses have provided insight on the number of stages that are 372 involved in the thermal decomposition of PI. Using knowledge acquired from the 373 thermal decomposition of $\mathrm{PI}$ in our previous work ${ }^{2}$, kinetic model for the thermal decomposition of PI under $2 \%$ oxygen is suggested (Scheme 2).

Scheme 2. Kinetic model used to model the thermal decomposition kinetics of PI under $2 \%$ oxygen

376 Similarly to the decomposition of PI under nitrogen, a three-stage pathway was used 377 to model the thermal decomposition of PI under $2 \%$ oxygen. However, in order to 378 involve the thermo-oxidation reactions in the model, a supplementary stage was 379 required. From the TGA of PI under varying oxygen concentrations ${ }^{2}$, it was seen that 380 oxygen intervenes right at the start of the decomposition. Therefore, the thermo- 
oxidation stage was added as a competitive reaction at the very first stage of the decomposition model (stage 1.2).

383 The optimized kinetic parameters for the thermal decomposition of PI under $2 \%$ 384 oxygen are summarized in Table 3.

Table 3. Kinetic parameters used in the model for the thermal decomposition of PI under $2 \%$ oxygen

The optimized parameters for the kinetic model fit well with the experimental TG curves (Figure 12). The activation energy for the two competitive reactions at the beginning of the reaction are of the same order of magnitude. One of the two reactions from the first stage of the decomposition is assigned to the thermal decomposition of $\mathrm{PI}$, while the other one is assigned to the thermo-oxidation of the polymer backbone.

Since thermo-oxidation usually results in the formation of carbon dioxide, carbon monoxide and water, no subsequent reaction is assigned after the reaction corresponding to the thermo-oxidation of the polymer backbone.

The second stage is assigned to the subsequent thermal decomposition of the decomposition products formed during the initial thermal decomposition. Indeed, from the previous study, we have concluded that the first decomposition of PI may lead to the formation of a thermally stable char, or another crosslinked structure.

The formation of this structure is aided by the presence of radicals resulting from the initial decomposition reactions (stage 1.1). Moreover, this structure may further thermally decompose into smaller structures. This leads to the formation of more radicals that can in turn participate in furthering the decomposition. Therefore, the second stage of the decomposition of PI was assigned to an autocatalytic reaction type. This stage has a relatively low activation energy because of the presence of radicals that are still lingering around the polymer after the first decomposition stage. Moreover, these recombination reactions eventually lead to stable char formation as it was seen in our previous work dealing with the decomposition mechanism of $\mathrm{PI} .{ }^{12}$ Char formation reactions are exothermic and could also explain the lowering of the activation energy on the activation energy plot on Figure $10 .{ }^{13}$

The last reaction stage corresponds to the thermo-oxidation of the remaining organic material and carbonaceous char.

Figure 12. Best fit of the TG data for the three-stage reaction models in Scheme 2, with the parameters given on Table 3. The curves represent the experimental data and the symbols represent the model.

When comparing with the decomposition model under nitrogen, an additional competitive decomposition reaction is observed in the first stage of the thermal decomposition model of $\mathrm{PI}$ under low oxygen concentration. This is because thermo- 
oxidation occurs right at the beginning of the decomposition of PI, parallelly to 419 pyrolytic decomposition. It is interesting to note that these two initial decomposition reactions have a significant contribution to the decomposition. Indeed, about $50 \%$ of the model is assigned to the two initial stages. The other decomposition stages correspond to the char degradation and oxidation. Moreover, from a statistical point of view, the correlation coefficient between the experimental curve and the simulated curve based on the kinetic model is 0.99949., meaning that the model used to simulate the thermal decomposition of $\mathrm{PI}$ under $2 \%$ oxygen is consistent with the experimental TG curves.

To complete the investigation of the kinetics of the thermal decomposition of PI under different oxygen concentrations, the behavior of $\mathrm{PI}$ in air was also investigated.

\subsection{PI decomposition in air}

After having investigated the thermal decomposition behavior of PI under low oxygen concentrations the kinetic of the thermal decomposition of PI in air was studied so that the effect of a highly thermo-oxidative atmosphere can be better understood. This also allows for the simulation of the degradation behavior when the material is subjected to heat in a well-ventilated environment. The presence of oxygen in air usually increases the extent to which a material decomposes when under thermal stress. Sometimes, oxygen can have a catalytic effect on the onset of the decomposition of a material.

Dynamic TGA was used to investigate the kinetics of the thermal decomposition behavior of $\mathrm{PI}$ in air. The TG curves of PI and their corresponding DTG curves at four different heating rates are shown on Figure 13.

443 From the TG curves of $\mathrm{PI}$ in air, it can be seen that the thermal stability of $\mathrm{PI}$ is maintained until around $450{ }^{\circ} \mathrm{C}$, whereby the onset of thermal decomposition is observed at the beginning of a mass loss process. The TG curves all appear to follow an apparent single stage. However, on the DTG curves, at high heating rates, a second peak, which is close to the first, is noticeable. This means that the kinetics of the thermal decomposition of PI in air occurs in at least two stages. In order to obtain further information regarding the number of stages and the types of reactions occurring during the thermo-oxidative decomposition of $\mathrm{PI}$ in air, the Friedman plot for the corresponding heating rates was plotted (Figure 14). 
454 From the Friedman plot, an accelerated decomposition stage can be distinguished 455 as a first peak. The peak slope is steeper than the isoconversion lines. This suggests 456 that the first decomposition stage is accelerated, probably autocatalytic ${ }^{9}$. At higher 457 heating rates $(5$ and $10 \mathrm{~K} / \mathrm{min}$ ) there seems to be another peak that is visible right 458 after the first one. This confirms that there are at least two stages in the thermal 459 decomposition of $\mathrm{PI}$ in air. it is therefore likely that at low heating rates, the two 460 thermal decomposition stages are overlapping.

461 In order to see any eventual reactions that may be occurring during the thermo462 oxidative decomposition of $\mathrm{PI}$, the activation energy and log (pre-exponential factor) 463 (calculated from the Friedman analysis), was plotted with respect to the conversion 464 (Figure 15).

Figure 15. Activation energy plot for PI in air, obtained by Friedman analysis

The activation energy is relatively high at the beginning of the conversion (around $190 \mathrm{~kJ} / \mathrm{mol}$ ). It decreases until around $60 \%$ conversion where the activation energy is at around $130 \mathrm{~kJ} / \mathrm{mol}$. This means that there is another stage in the decomposition of $\mathrm{PI}$. At this point, and until almost $98 \%$ conversion, there is little evolution in the activation energy. At $100 \%$ conversion, there is a high increase in activation energy. However, the error at this conversion is very high, therefore, it was not taken into

473 The activation energy plot based on the Friedman method provided out the number 474 of consecutive stages occurring during the thermo-oxidative decomposition of the material. To further the understanding of decomposition in terms of competitive reactions that may occur, the activation energy plot based on the Friedman method was compared with that based on the OFW method (Figure 16).

Figure 16. Activation energies calculated using Friedman analysis (black) and the Ozawa-Flynn-Wall method (magenta) for $\mathrm{PI}$ in air.

480 From the two activation energy plots on Figure 16, it can be seen that the two 481 methods lead to very different activation energies in at around $50 \%$ conversion. This strongly suggests that there are competitive reactions occurring during the thermal decomposition of $\mathrm{PI}$ in air. It was therefore considered when elaborating the kinetic model for the thermo-oxidative decomposition of PI.

485 Based on knowledge acquired during the investigation of the thermal stability of PI and its thermal decomposition mechanism under nitrogen, we were able to take an attempt at defining a kinetic model for the thermo-oxidative decomposition of $\mathrm{PI}$ in air. The model is shown on Scheme 3. 
490 From Scheme 3, two major consecutive decomposition stages are illustrated. The 491 first corresponds to the initial decomposition of PI, similarly to the thermal 492 decomposition of PI under nitrogen. It corresponds to the random scission of the 493 polymer backbone. However, it was observed that the presence of oxygen tends to 494 slightly decrease the thermal stability of $\mathrm{PI}$ at high temperatures ${ }^{2}$. This translates into 495 a lower activation energy for the onset of the decomposition. Additionally, the initial 496 decomposition leads to the formation of reactive radicals which can take part in the 497 scission of the initial polymer. This effect can be assigned to an autocatalytic type of 498 decomposition reaction whereby the products increase the rate of the 499 decomposition. During this stage, rearrangement of the polymer backbone to form a 500 crosslinked char structure was also hypothesized under nitrogen. This char is highly 501 stable under inert atmosphere, but not under oxygen. This was evidenced by the 502 TGA under different oxygen levels, whereby the second decomposition of PI was 503 largely affected by the presence of oxygen.

504 It was therefore not unexpected to add a stage whereby the thermo-oxidative 505 reactions are occurring. This corresponds to stage 2.2. Furthermore, while the 506 material is being thermo-oxidized, there are also thermal decomposition that is on507 going, via a similar mechanism as the first stage. However, the initial structure of the 508 polymer has changed, and so have the kinetic parameters for the decomposition 509 stage. Therefore, an autocatalytic type decomposition mechanism was assigned to 510 this stage.

511 The optimized kinetic parameters used in for the modeling of the thermal 512 decomposition of PI in air are summarized in Table 4.

Table 4. Kinetic parameters used in the model for the thermal decomposition of PI in air

514 The activation energy for the first decomposition stage is coherent with the activation 515 energy calculated using the Friedman analysis for the first stage (Figure 15). This 516 first stage of thermal decomposition formed a charred structure and possibly a 517 partially decomposed form of the polymer. Further thermal decomposition of this 518 structure is modeled by reaction 2.1. This was assigned to a similar reaction as the 519 first decomposition stage. However, since the chains have already partially been 520 broken, the activation energy is lower. Moreover, in the presence of air, the structure 521 undergoes thermo-oxidation (modeled by reaction 2.2).

522 The simulated TG curves based on the kinetic model above as well as the 523 experimental TG curves are shown on Figure 17.

Figure 17. Best fit of the TG data for the three-stage reaction models in Scheme 3, with the parameters given on Table 4. The curves represent the experimental data and the symbols represent the model. 
Contrarily to the kinetic model of the thermal decomposition of PI under $2 \%$ oxygen, the model in air does not have competitive reactions in the initial decomposition stage. However, the autocatalytic nature of the reaction model suggests that there are reactive decomposition products that are released during that stage, leading to the formation of a char as well as releasing oxidation products. Moreover, the other two decomposition reactions have been assigned to two thermo-oxidation reactions, each with a significant contribution as compared to the first stage of the decomposition. Indeed, the decomposition of the char (stage 2.2) and the further thermo-oxidation of the polymer (stage 2.1) play a bigger role in the thermal decomposition than the initial char formation reaction (stage 1). It is interesting to note that the thermo-oxidation of the char corresponds to a low order kinetic reaction model (reaction order $=0.06$ ). This means that it is only slightly dependent on the "concentration" of the reactants.

The fitted curve seems to correspond well with the experimental curve. From a statistical point of view, the correlation coefficient between the experimental and the simulated curve is 0.99993 .

\section{Discussion and Conclusion}

544 The kinetic parameters for the thermal decomposition of PI under three different 545 oxygen levels have been calculated. We have seen that, from a kinetic perspective, the decomposition pathway adopted by PI during its thermal decomposition is significantly dependent on the oxygen concentration.

548 Under nitrogen, the kinetic pathway adopted during its thermal decomposition is relatively simple. However, the presence of a small amount of oxygen brings about a significant change in kinetic pathway adopted by the material during its thermal decomposition. Indeed, in the presence of a small concentration of oxygen, the decomposition pathway is much complexified, leading to a 3-stage decomposition involving a competitive reaction at the initial decomposition stage. Moreover, it was reported that the presence of oxygen increases the crosslinking that occurs in a material ${ }^{14}$. This can be extrapolated to $\mathrm{PI}$, which is also a highly charring polymeric material. Therefore, the higher activation energy that is observed for the thermal decomposition of $\mathrm{PI}$ under low oxygen concentration suggests that major crosslinking would have had occurred during the initial decomposition reactions.

Under air, the activation energy for the onset of the decomposition is lower than under pyrolytic conditions. One explanation for this can be that decomposition gases that are produced at the beginning of the decomposition are readily oxidized. A fast consumption of the decomposition gases pulls the kinetics towards the further decomposition of the material. Moreover, the presence of recombination reactions within the polymeric matrix may also be occurring owing to the charring properties of 
565 the polymer can also explain the lowered activation energy due to their exothermic 566 nature.

567 We have seen that from a kinetics perspective, the pathway adopted by PI during its 568 thermal decomposition is highly dependent on the oxygen concentration. Moreover, 569 low oxygen concentration leads to a higher crosslinking, leading to an increase in 570 the activation energy of the onset of decomposition. However, in air, the crosslinking 571 is taken over (for the most part), by thermo-oxidation. The difference in the kinetics 572 of the thermal decomposition of PI has provided deep insight on the eventual 573 behavior of the material under a fire scenario.

574

575 Funding: This work has received funding from the European Research Council's 576 under the European Union's H2020 - the Framework programme for Research and 577 Innovation (2014 - 2020) / ERC Grant Agreement No. 670747 - ERC 2014 578 AdG/FireBar-Concept

579 Conflicts of Interest: The authors declare no conflict of interest. 


\section{References}

583 1. Ramgobin A, Fontaine G, Bourbigot S. Thermal Degradation and Fire Behavior of High Performance Polymers. Polym Rev. 2019;59(1):55-123. doi:10.1080/15583724.2018.1546736

2. Ramgobin A, Fontaine G, Bourbigot S. Investigation of the thermal stability and fire behavior of high performance polymer: A case study of polyimide. Fire Saf J. 2020;(April):103060. doi:10.1016/j.firesaf.2020.103060

3. Opfermann J. Kinetic analysis using multivariate non-linear regression. I. Basic concepts. J Therm

4. Kaisersberger E, Opfermann J. Kinetic evaluation of exothermal reactions measured by DSC. Thermochim Acta. 1991;187(C):151-158. doi:10.1016/0040-6031(91)87189-4

5. Moukhina E. Determination of kinetic mechanisms for reactions measured with

6. Kinetics SR. Chapter 3 Theory of Solid State Reaction Kinetics. Compr Chem thermoanalytical instruments. J Therm Anal Calorim. 2012;109(3):1203-1214. doi:10.1007/s10973-012-2406-3 Kinet. 1980;22(C):41-113. doi:10.1016/S0069-8040(08)70384-4

7. Vyazovkin S. Kinetic concepts of thermally stimulated reactions in solids: A view from a historical perspective. Int Rev Phys Chem. 2000;19(1):45-60. doi:10.1080/014423500229855

9. Hondred PR, Yoon S, Bowler N, Moukhina E, Kessler MR. Degradation

10. Ozawa T. A New Method of Analyzing Thermogravimetric Data. Bull Chem Soc Jpn. 1965;38(11):1881-1886. doi:10.1246/bcsj.38.1881

11. Flynn JH, Wall LA. A quick, direct method for the determination of activation energy from thermogravimetric data. J Polym Sci Part B Polym Lett. 1966;4(5):323-328. doi:10.1002/pol.1966.110040504

12. Ramgobin A, Fontaine $G$, Bourbigot $S$. Investigation of the thermal stability and fire behavior of high performance polymer: A case study of polyimide. Fire Saf J. 2020;(May). doi:10.1016/j.firesaf.2020.103060

13. Li J, Stoliarov SI. Measurement of kinetics and thermodynamics of the thermal degradation for charring polymers. Polym Degrad Stab. 2014;106:2-15. 
628

629

\section{Figures}

Figure 1. TG and DTG plots of PI heated at $10{ }^{\circ} \mathrm{C} / \mathrm{min}$ under nitrogen, $2 \%, 4 \%, 8 \%$ and $12 \%$, and in air².

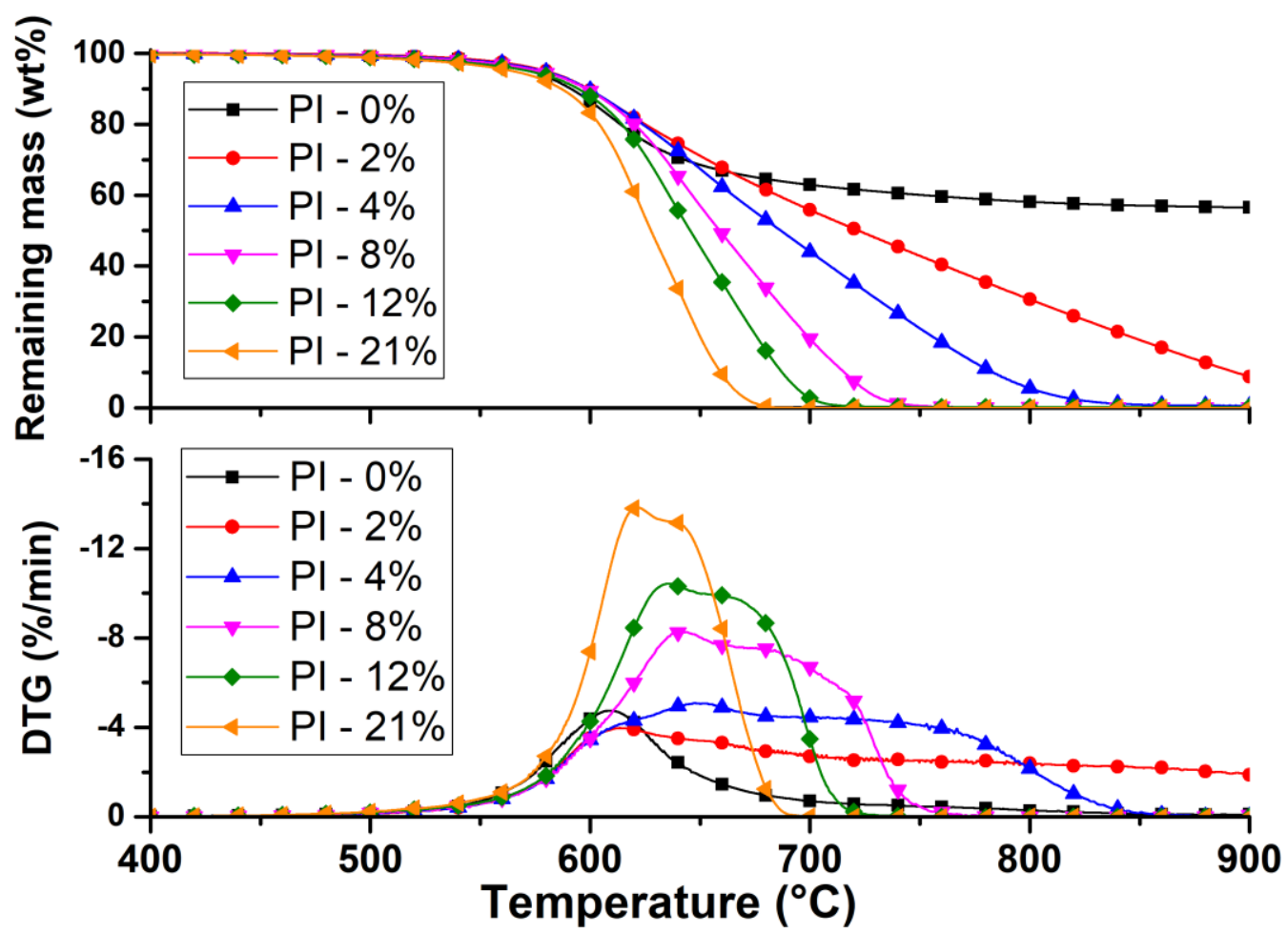

Figure 2. Friedman plots corresponding to a normal type of reaction (left), an accelerated reaction (middle), and a retarded reaction (right). ${ }^{9}$
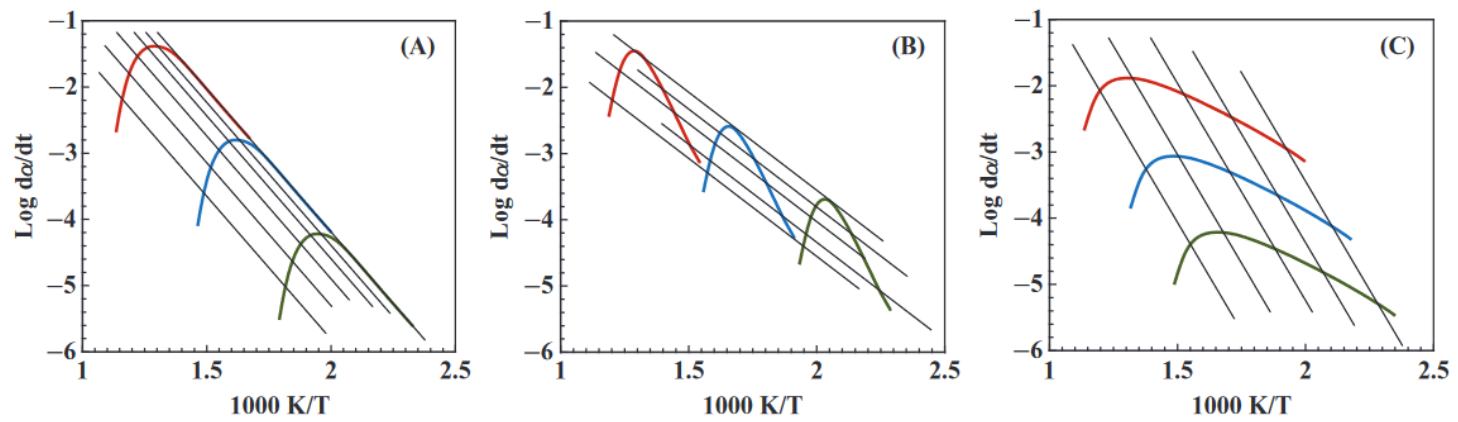
633 Figure 3. TGA (left) and the corresponding DTG (right) curve of PI at 1 (black), 2 634 (red), 5 (blue), and $10 \mathrm{~K} / \mathrm{min}$ (magenta), under nitrogen.
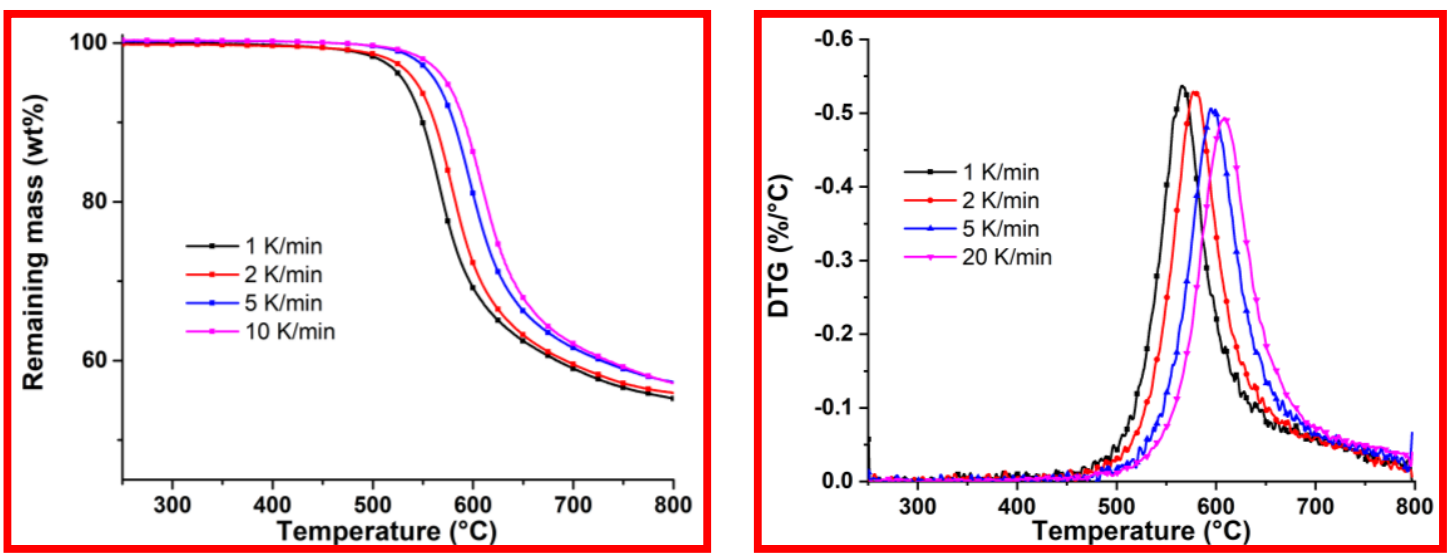

636

637 Figure 4. Friedman analysis of $\mathrm{PI}$ using 4 different heating rates under nitrogen 638 (orange: $1 \mathrm{~K} / \mathrm{min}$, green: $2 \mathrm{~K} / \mathrm{min}$, red: $5 \mathrm{~K} / \mathrm{min}$, blue: $10 \mathrm{~K} / \mathrm{min}$ )

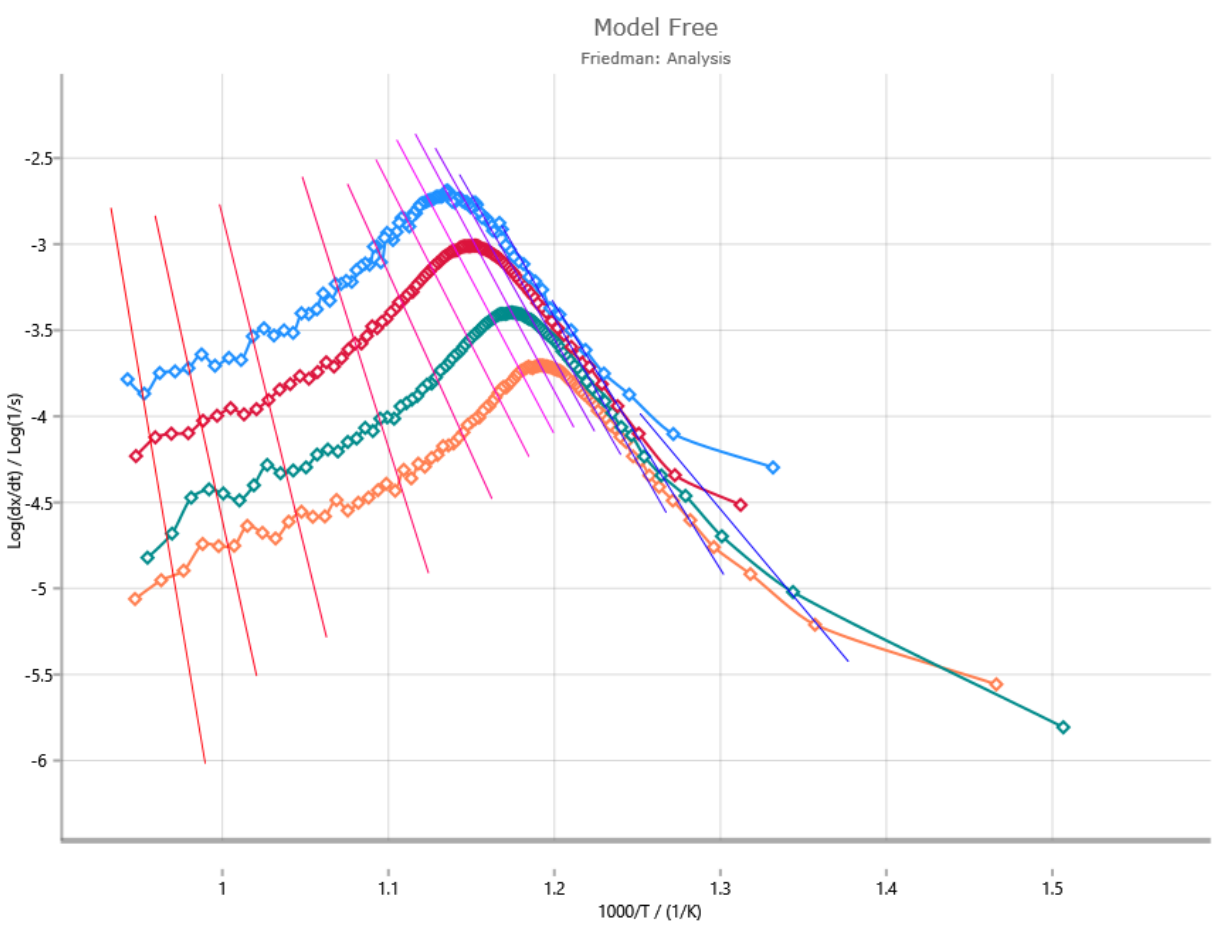


641 Figure 5. Activation energy plot for PI under nitrogen (left), obtained by Friedman 642 analysis

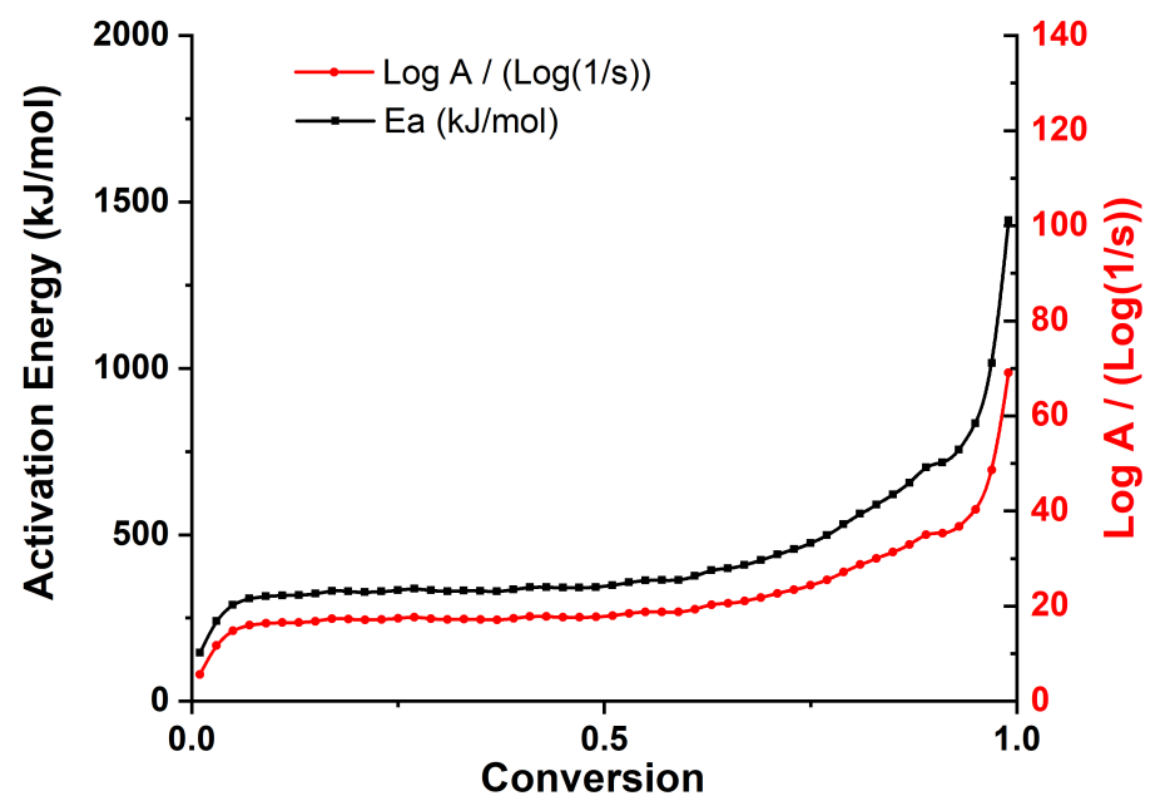

643

644 Figure 6. Activation energies calculated using Friedman analysis (black) and the 645 Ozawa-Flynn-Wall method (magenta) for PI under nitrogen

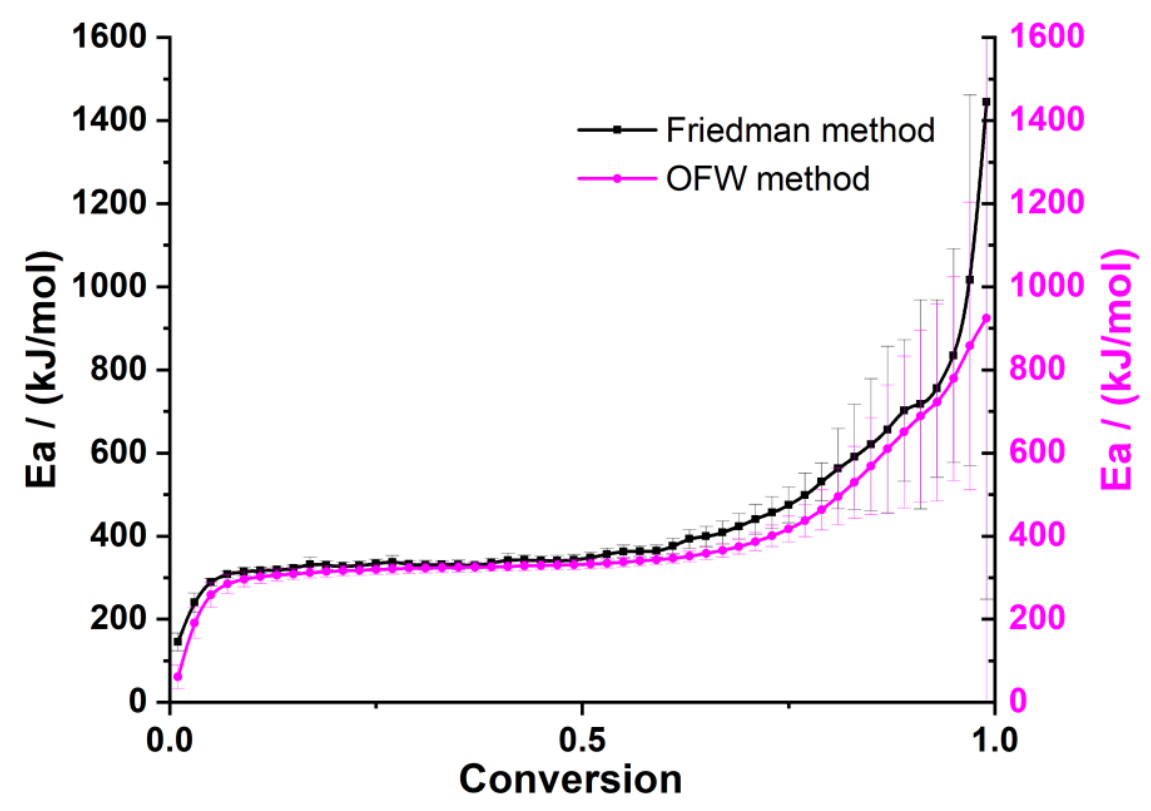

646 
648 Figure 7. Best fit of the TG data for the three-stage reaction models in Scheme 1, 649 with the parameters given on Scheme 1. The curves represent the experimental 650 data and the symbols represent the model

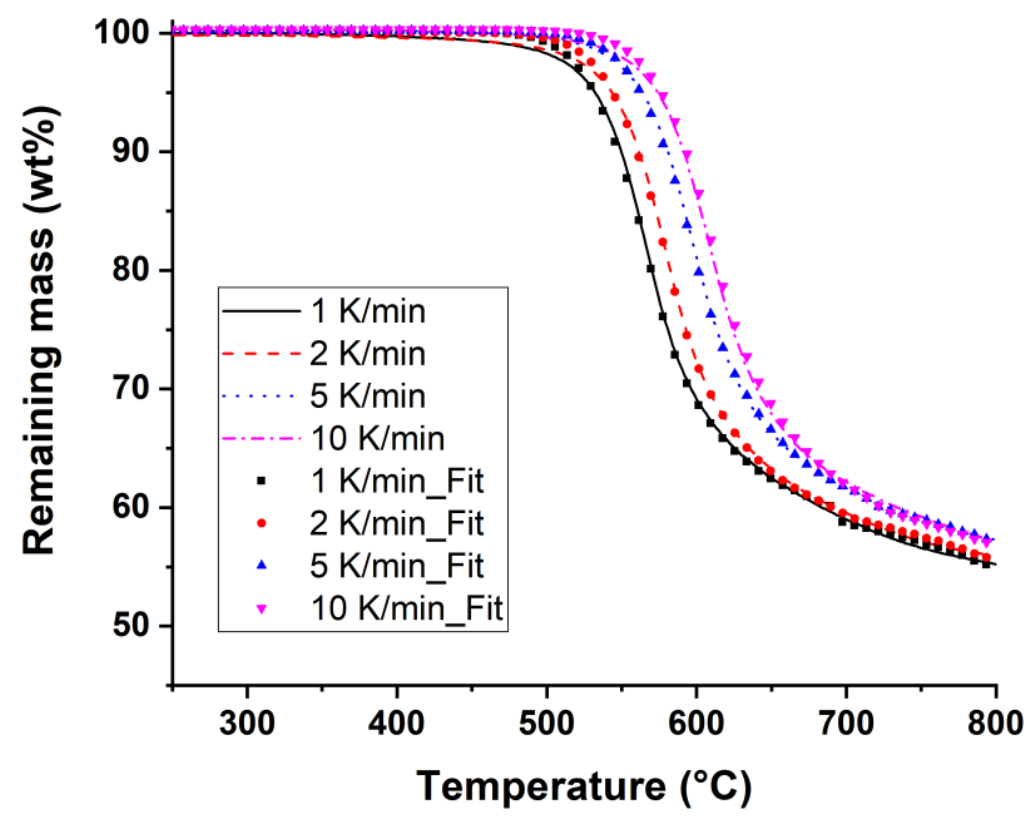

652 Figure 8. TGA (left) and the corresponding DTG (right) curve of PI at 1 (black), 2 653 (red) ,5 (blue), and $10 \mathrm{~K} / \mathrm{min}$ (magenta), under $2 \%$ oxygen

654
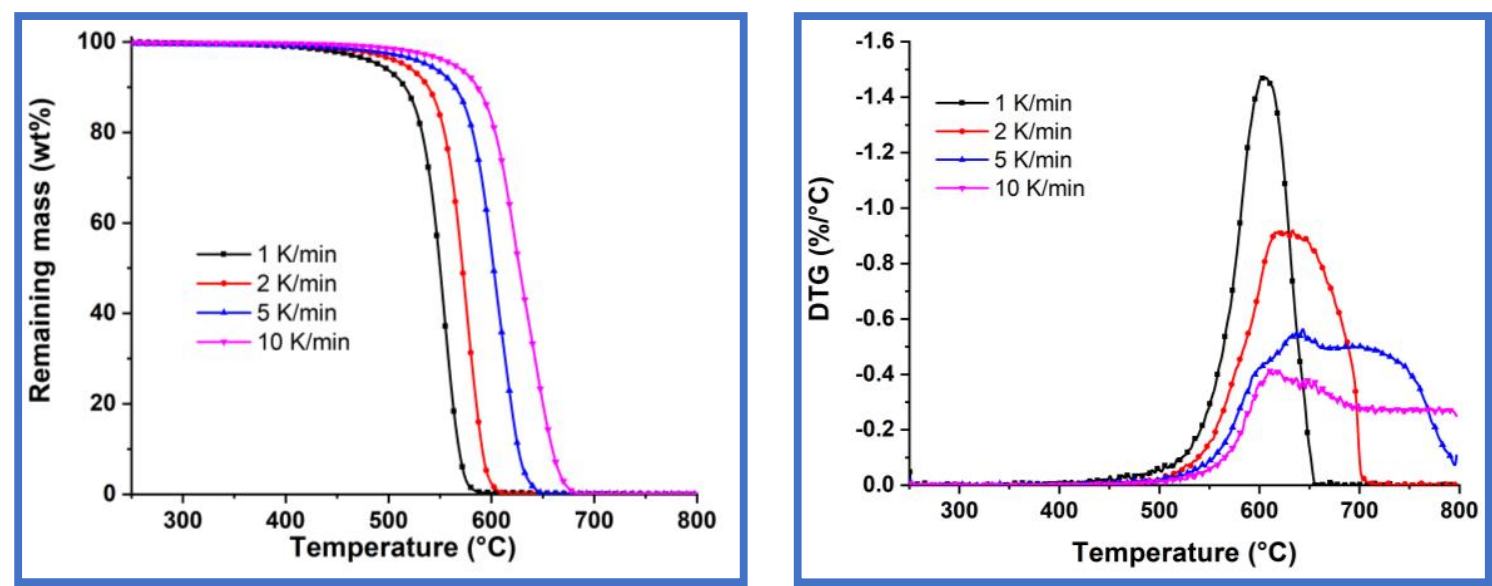
657 Figure 9. Friedman analysis of PI using 4 different heating rates under $2 \%$ oxygen 658 (orange: $1 \mathrm{~K} / \mathrm{min}$, green: $2 \mathrm{~K} / \mathrm{min}$, red: $5 \mathrm{~K} / \mathrm{min}$, blue: $10 \mathrm{~K} / \mathrm{min}$ )

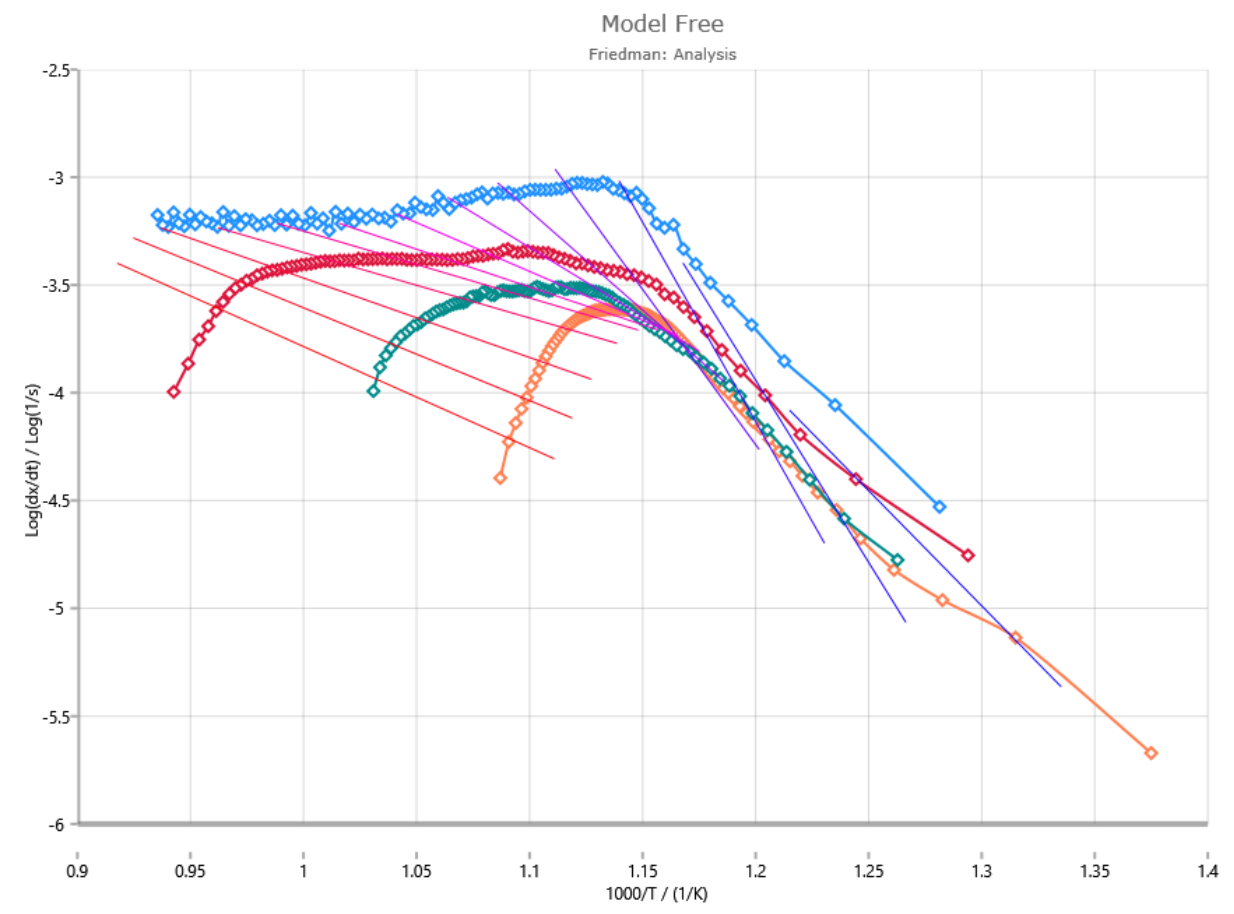

660 Figure 10. Activation energy plot for PI under 2\% oxygen, obtained by Friedman 661 analysis

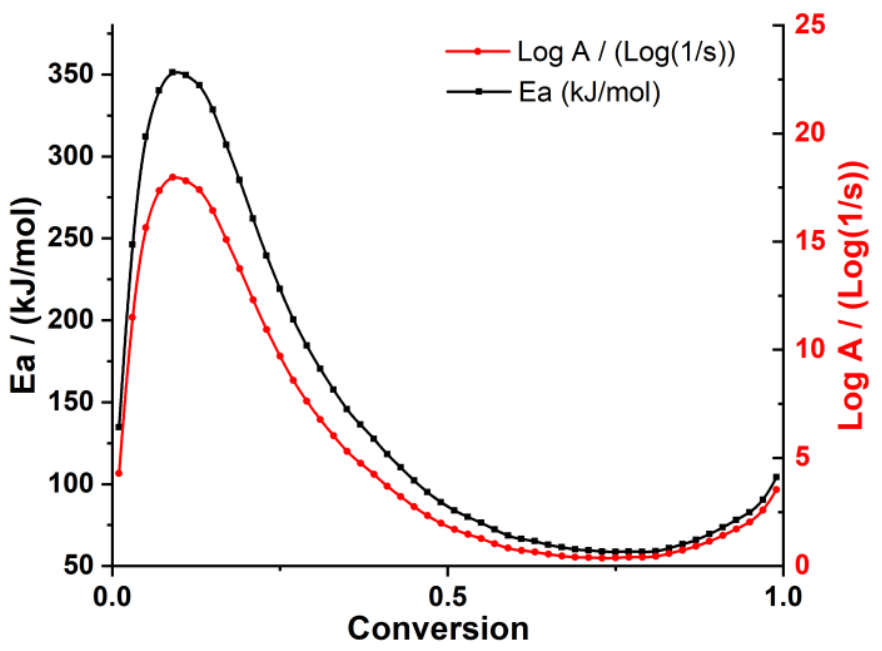

662 
664 Figure 11. Activation energies calculated using Friedman analysis (black) and the 665 Ozawa-Flynn-Wall method (magenta) for PI under 2\% oxygen

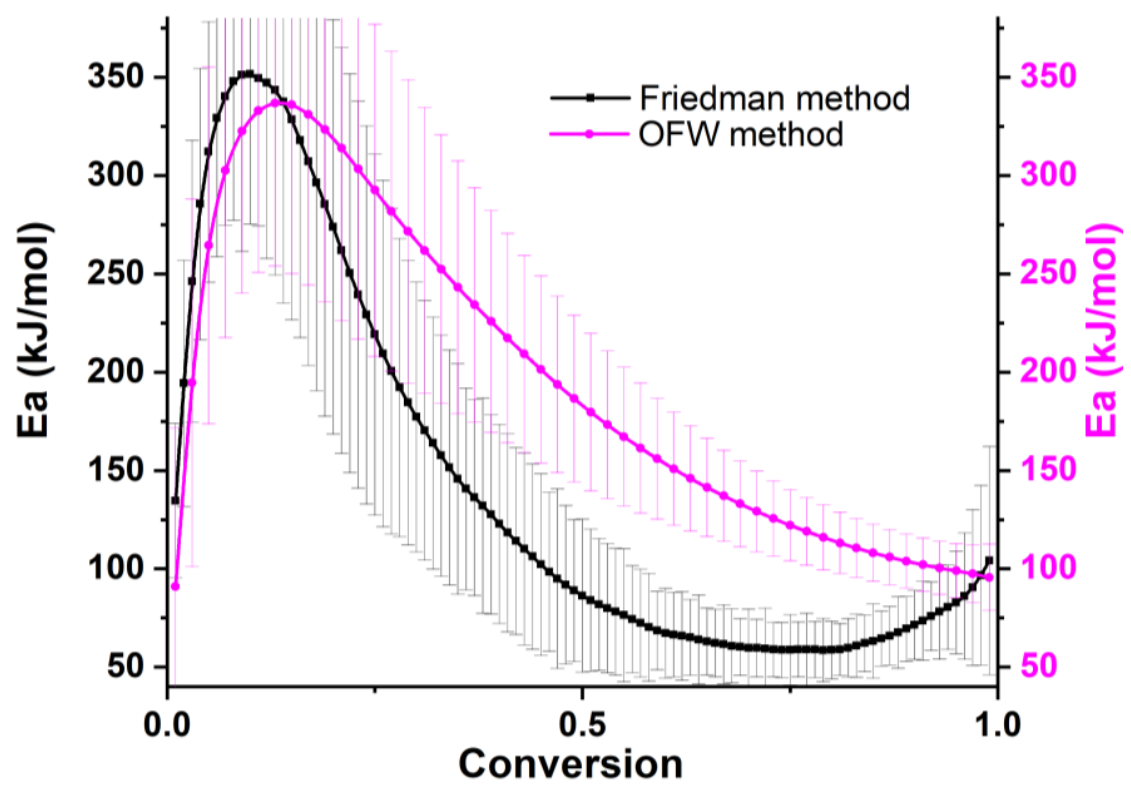

666

667 Figure 12. Best fit of the TG data for the three-stage reaction models in Scheme 2, 668 with the parameters given on Table 3. The curves represent the experimental data 669 and the symbols represent the model.

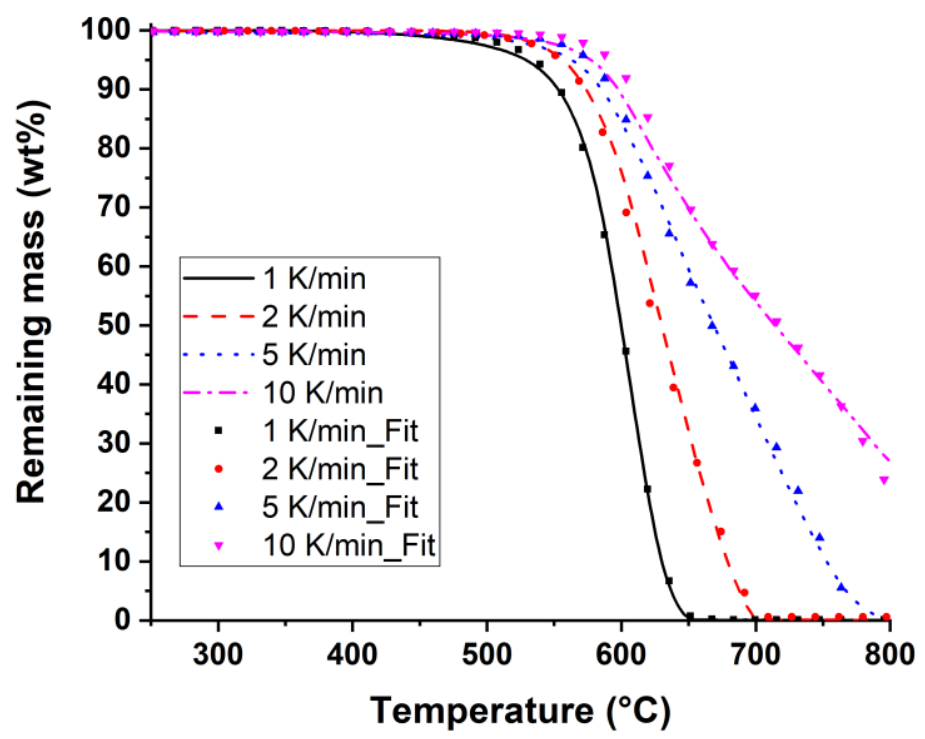

670 
672 Figure 13. TGA (left) and the corresponding DTG (right) curve of PI at 1 (black), 2 673 (red), 5 (blue), and $10 \mathrm{~K} / \mathrm{min}$ (magenta), in air.
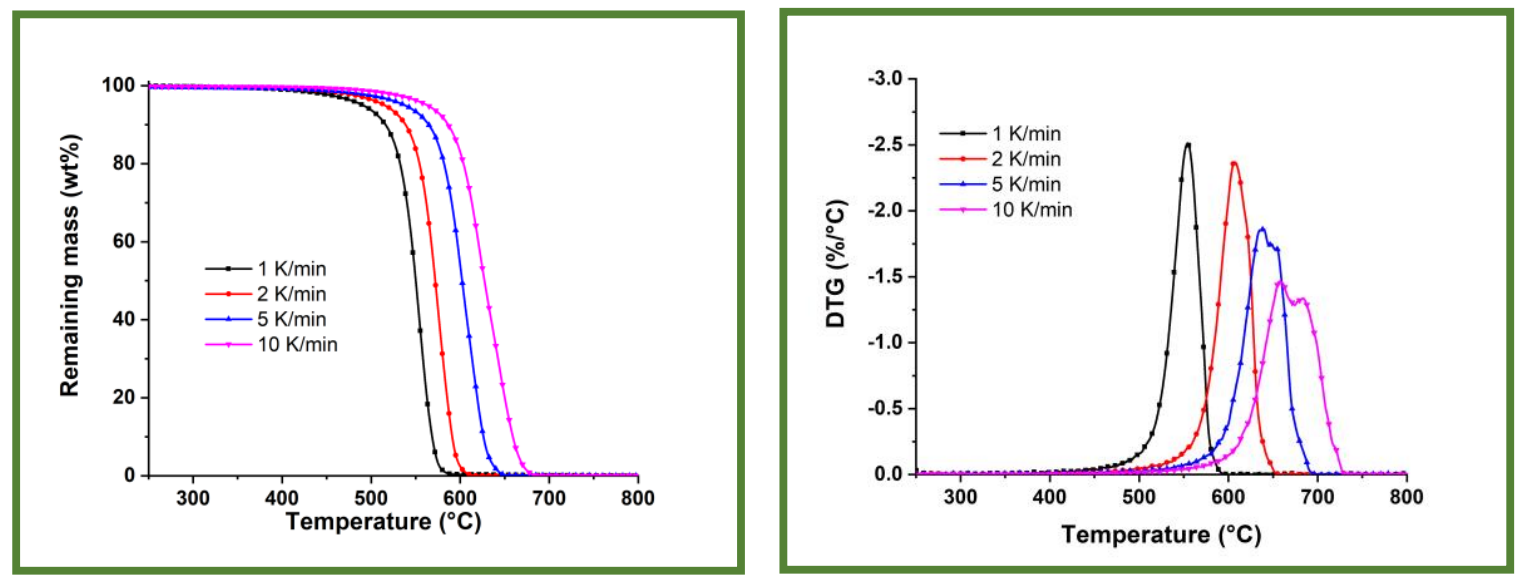

674

675 Figure 14. Friedman analysis of PI using 4 different heating rates in air (orange: 1 $676 \mathrm{~K} / \mathrm{min}$, green: $2 \mathrm{~K} / \mathrm{min}$, red: $5 \mathrm{~K} / \mathrm{min}$, blue: $10 \mathrm{~K} / \mathrm{min}$ )

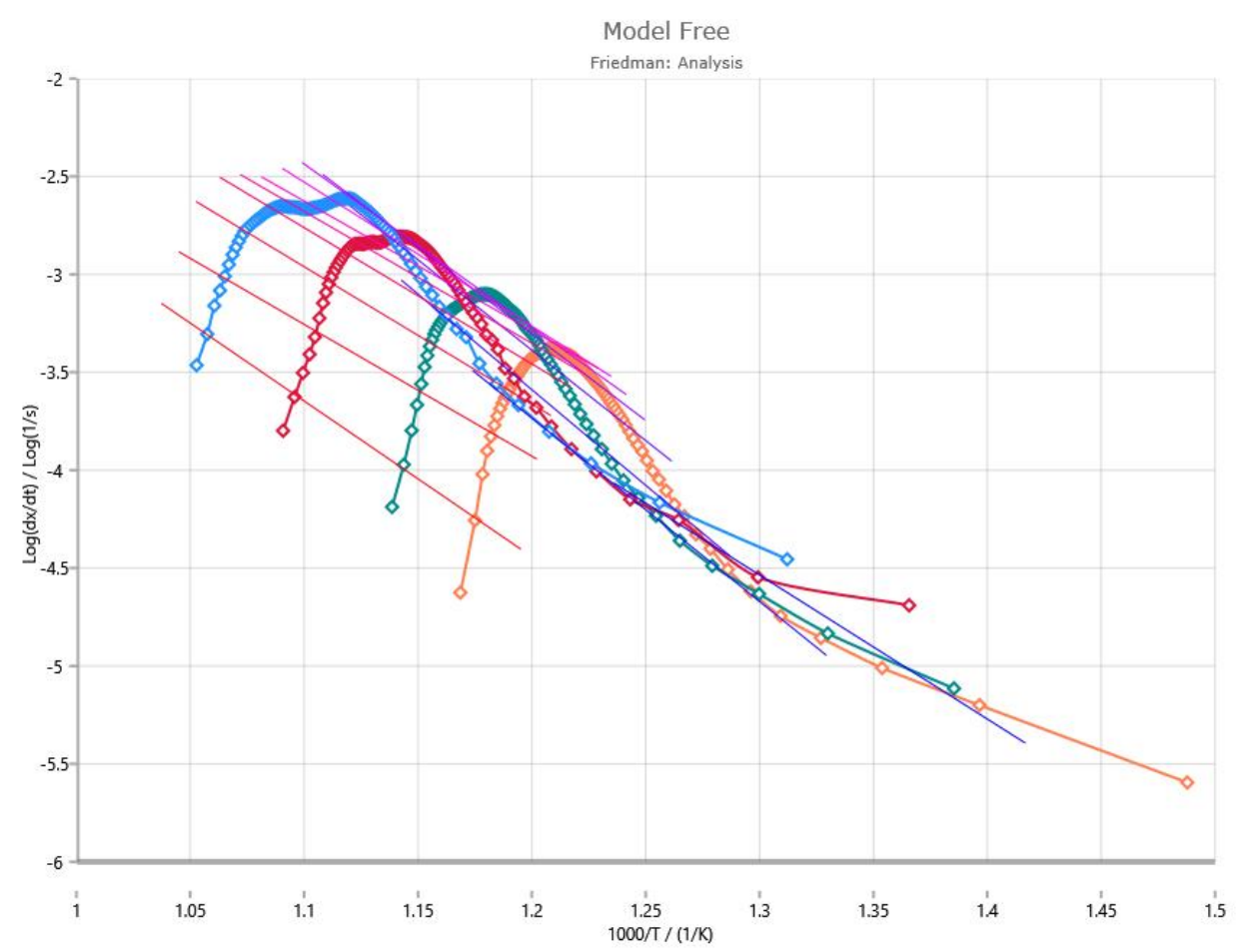

677

678 
679 Figure 15. Activation energy plot for PI in air, obtained by Friedman analysis

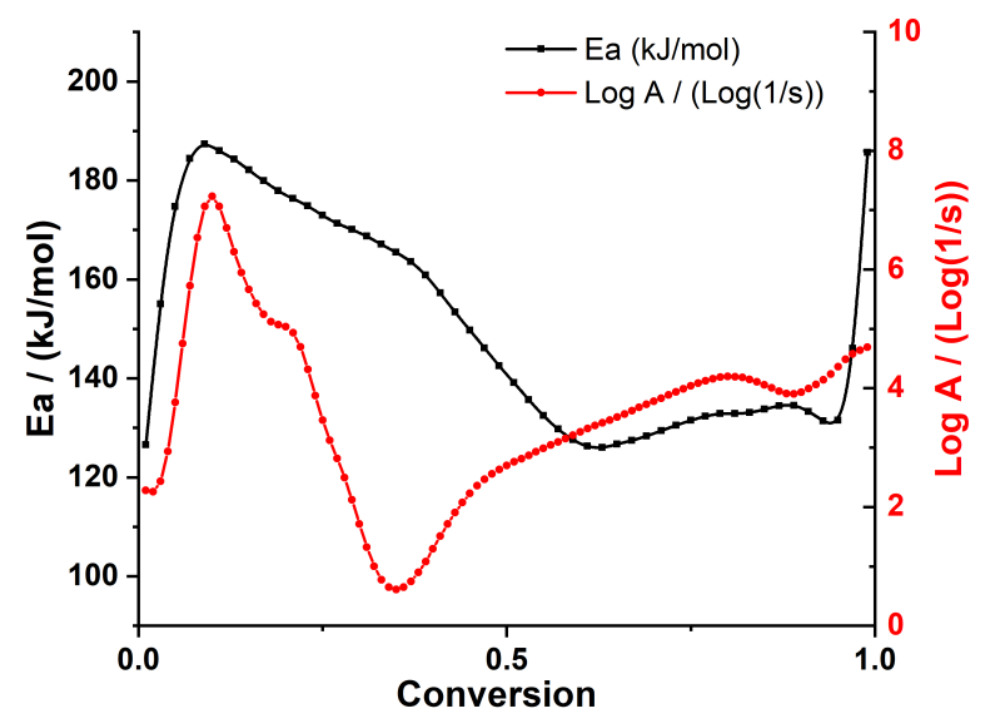

680

681 Figure 16. Activation energies calculated using Friedman analysis (black) and the 682 Ozawa-Flynn-Wall method (magenta) for PI in air.

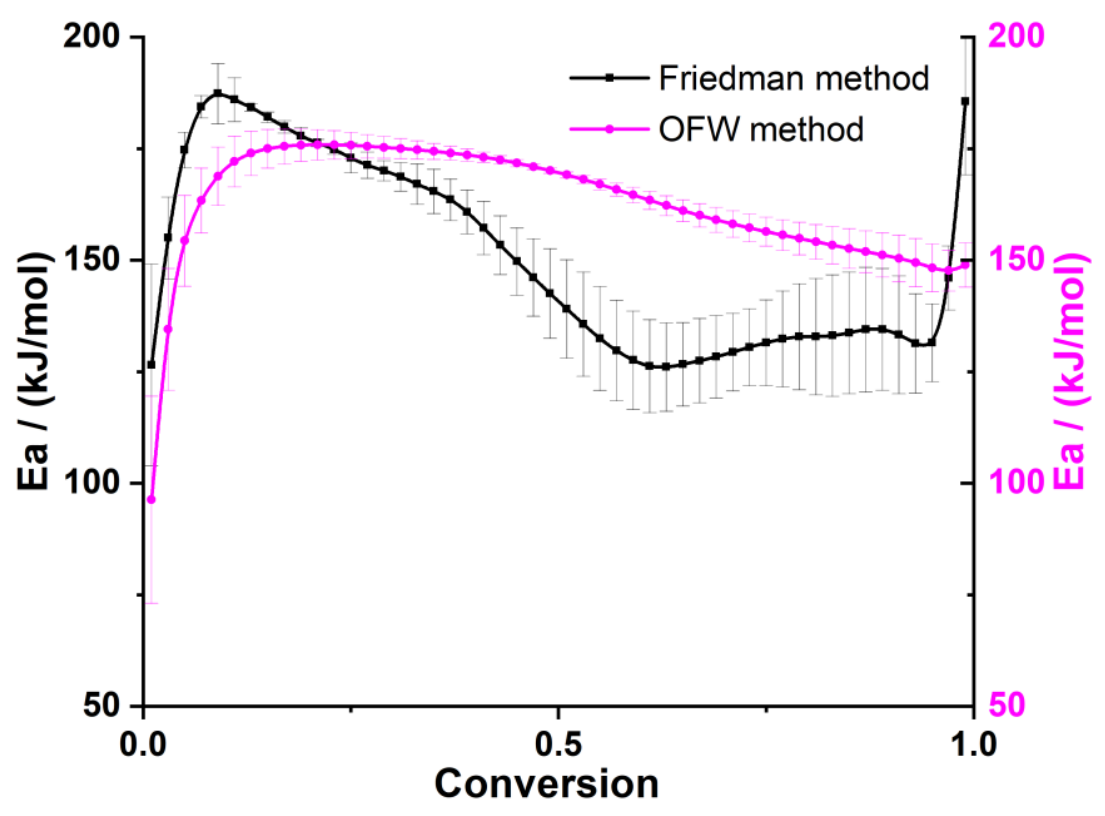

683 
685 Figure 17. Best fit of the TG data for the three-stage reaction models in Scheme 3, 686 with the parameters given on Table 4. The curves represent the experimental data 687 and the symbols represent the model.

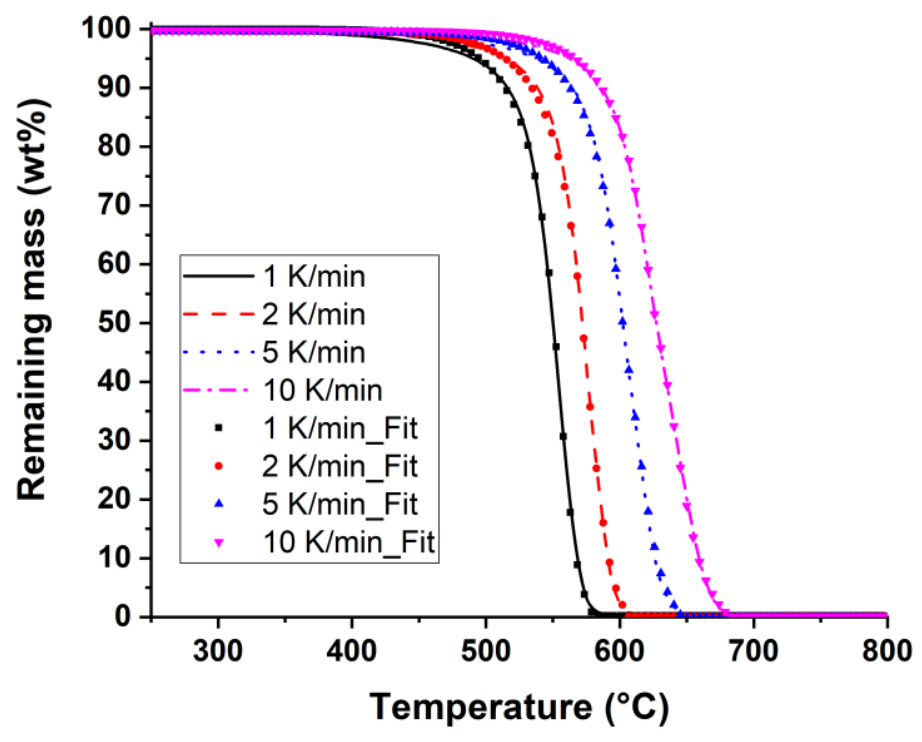

688

689 
690 Scheme 1. Kinetic model used to model the thermal decomposition kinetics of PI 691 under nitrogen.

692

693

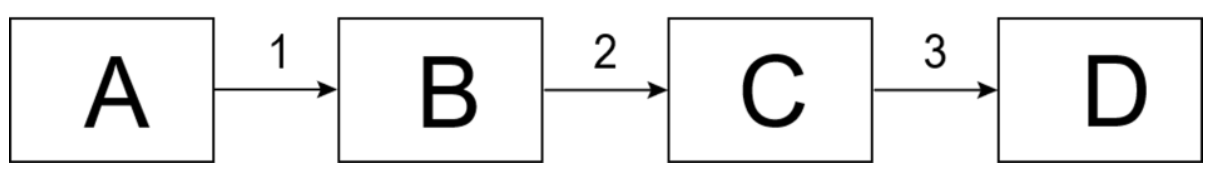

694

695 Scheme 2. Kinetic model used to model the thermal decomposition kinetics of PI 696 under $2 \%$ oxygen

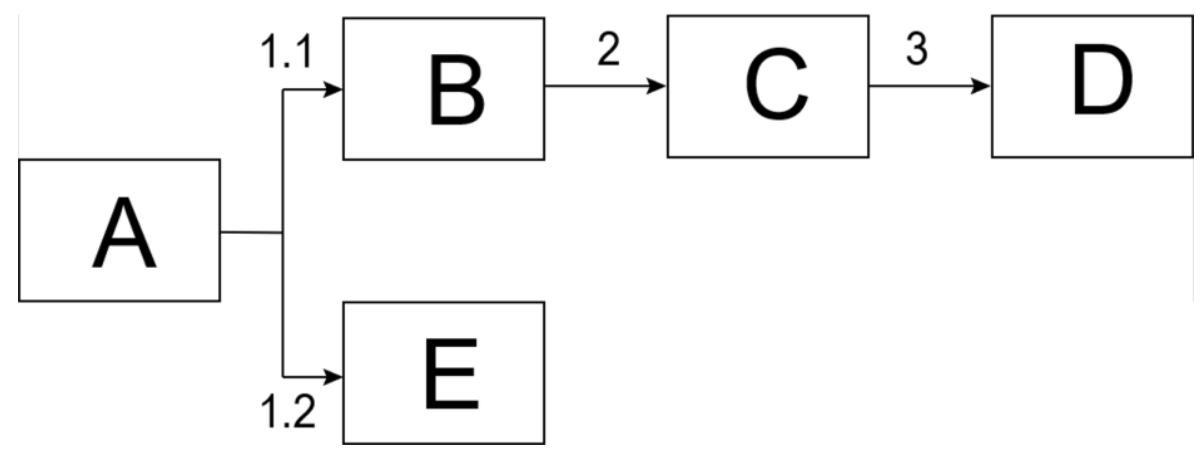

698

699 Scheme 3. Kinetic model used to model the thermal decomposition kinetics of PI in 700 air.

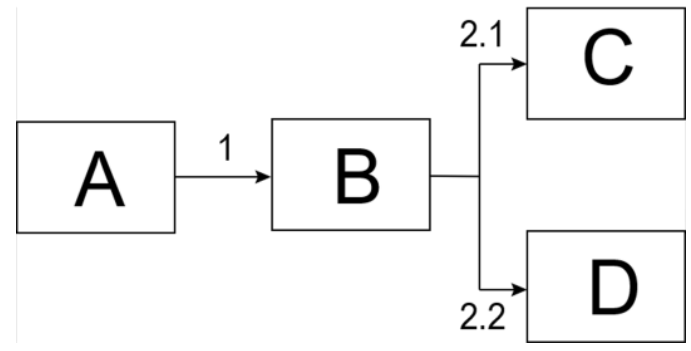


Table 1. Reaction types and corresponding reaction equations $d \alpha d t=$ $704-A \operatorname{eERTf}(\alpha)$

\begin{tabular}{|c|c|c|}
\hline Code & Function & Type of reaction \\
\hline $\mathrm{F} 1$ & $f=(1-\alpha)$ & Reaction of 1 st order \\
\hline $\mathrm{F} 2$ & $f=(1-\alpha)^{2}$ & Reaction of 2 nd order \\
\hline Fn & $f=(1-\alpha)^{n}$ & Reaction of nth order \\
\hline R2 & $f=2(1-\alpha)^{\frac{1}{2}}$ & Two-dimensional phase boundary \\
\hline R3 & $f=3(1-\alpha)^{\frac{2}{3}}$ & Three-dimensional phase boundary \\
\hline D1 & $\mathrm{f}=\frac{1}{2} \cdot \frac{1}{\alpha}$ & One-dimensional diffusion \\
\hline D2 & $f=-\frac{1}{\ln ((1-\alpha))}$ & Two-dimensional diffusion \\
\hline D3 & $f=\frac{3}{2} \cdot \frac{(1-\alpha)^{\frac{2}{3}}}{1-(1-\alpha)^{\frac{1}{3}}}$ & Three-dimensional diffusion Jander's type \\
\hline D4 & $f=\frac{3}{2} \cdot \frac{1}{\left((1-\alpha)^{-\frac{1}{3}}-1\right)}$ & $\begin{array}{l}\text { Three-dimensional diffusion Ginstling- } \\
\text { Brounstein type }\end{array}$ \\
\hline B1 & $f=(1-\alpha) \cdot \alpha$ & Prout-Tompkins equation \\
\hline Bna & $\mathrm{f}=(1-\alpha)^{\mathrm{n}} \cdot \alpha^{\mathrm{K}_{\mathrm{cat}}}$ & Expanded Prout-Tompkins equation \\
\hline C1 & $\mathrm{f}=(1-\alpha) \cdot\left(1+\mathrm{K}_{\mathrm{cat}} \cdot \alpha\right)$ & $\begin{array}{l}\text { Reaction of } 1 \text { st order with autocatalysis by } \\
\text { product }\end{array}$ \\
\hline $\mathrm{Cn}$ & $\mathrm{f}=(1-\alpha)^{\mathrm{n}} \cdot\left(1+\mathrm{K}_{\mathrm{cat}} \cdot \alpha\right)$ & $\begin{array}{l}\text { Reaction of nth order with autocatalysis by } \\
\text { product }\end{array}$ \\
\hline Cnm & $\mathrm{f}=(1-\alpha)^{\wedge} \mathrm{n} \cdot\left(1+\mathrm{K}_{\mathrm{cat}} \cdot \alpha^{\mathrm{m}}\right)$ & $\begin{array}{l}\text { Reaction of nth order with m-Power } \\
\text { autocatalysis by product }\end{array}$ \\
\hline A2 & $f=2(1-\alpha) \cdot[-\ln (1-\alpha)]^{\frac{1}{2}}$ & $\begin{array}{l}\text { Two-dimensional nucleation according to } \\
\text { Avrami }\end{array}$ \\
\hline A3 & $f=3(1-\alpha) \cdot[-\ln (e)]^{\frac{2}{3}}$ & $\begin{array}{l}\text { Three-dimensional nucleation according } \\
\text { to Avrami }\end{array}$ \\
\hline An & $\mathrm{f}=\mathrm{n} \cdot(1-\alpha) \cdot[-\ln ((1-\alpha))]^{\frac{\mathrm{n}-1}{\mathrm{n}}}$ & $\begin{array}{l}\mathrm{n} \text {-dimensional nucleation according to } \\
\text { Avrami-Erofeev }\end{array}$ \\
\hline
\end{tabular}


706 Table 2. Kinetic parameters used in the model for the thermal decomposition of PI 707 under nitrogen

\begin{tabular}{l|lll}
\hline $\begin{array}{l}\text { Stage (reaction } \\
\text { type) }\end{array}$ & $1(\mathrm{Cna})$ & $2(\mathrm{Fn})$ & $3(\mathrm{Fn})$ \\
\hline Ea $(\mathrm{kJ} / \mathrm{mol})$ & 277.768 & 369.820 & 410.149 \\
$\log (\mathrm{A})(\log (1 / \mathrm{s})$ & 14.351 & 20.459 & 23.316 \\
Reaction order & 3.000 & 1.593 & 1.708 \\
Kcat & 0.377 & - & - \\
Contribution & 0.625 & 0.188 & 0.187 \\
\hline
\end{tabular}

708

709 Table 3. Kinetic parameters used in the model for the thermal decomposition of PI 710 under $2 \%$ oxygen

\begin{tabular}{l|llll}
\hline $\begin{array}{l}\text { Stage (reaction } \\
\text { type) }\end{array}$ & $1.1(\mathrm{Fn})$ & $1.2(\mathrm{Fn})$ & $2(\mathrm{Cn})$ & $3(\mathrm{Fn})$ \\
\hline Ea $(\mathrm{kJ} / \mathrm{mol})$ & 349 & 353 & 62 & 99 \\
$\log (\mathrm{A})(\log (1 / \mathrm{s})$ & 18.2 & 17.3 & 0.1 & 2.3 \\
Reaction order & 1.8 & 1.1 & 0.52 & 0.01 \\
Kcat & - & - & 1.69 & - \\
Contribution & 0.192 & 0.290 & 0.296 & 0.222 \\
\hline
\end{tabular}


714 Table 4. Kinetic parameters used in the model for the thermal decomposition of PI 715 in air

716

\begin{tabular}{l|lll}
\hline $\begin{array}{l}\text { Stage (reaction } \\
\text { type) }\end{array}$ & $\mathbf{1}(\mathrm{Cna})$ & $\mathbf{2 . 1}$ (Cna) & $\mathbf{2 . 2}$ (Fn) \\
\hline Ea $(\mathrm{kJ} / \mathrm{mol})$ & 181 & 122 & 112 \\
$\log (\mathrm{A})(\log (1 / \mathrm{s})$ & 7.9 & 4.2 & 2.9 \\
Reaction order & 0.41 & 1.24 & 0.06 \\
Kcat & 0.73 & 0.97 & - \\
Contribution & 0.166 & 0.291 & 0.543 \\
\hline
\end{tabular}

